\title{
Polinômios Ortogonais no Círculo Unitário com Relação a Certas Medidas Associadas a Coeficientes de Verblunsky Periódicos
}

\section{Cleonice F. Bracciali ${ }^{1}$}

Departamento de Matemática Aplicada, UNESP, São José do Rio Preto, SP Jairo S. Silva ${ }^{2}$

Pós-Graduação em Matemática, UNESP, São José do Rio Preto, SP

\section{A. Sri Ranga ${ }^{3}$}

Departamento de Matemática Aplicada, UNESP, São José do Rio Preto, SP

Daniel O. Veronese ${ }^{4}$

Pós-Graduação em Matemática, UNESP, São José do Rio Preto, SP

Resumo. É conhecido (veja [4]) que associado a um par de sequências reais $\left\{\left\{c_{n}\right\}_{n=1}^{\infty}\right.$, $\left.\left\{d_{n}\right\}_{n=1}^{\infty}\right\}$, com $\left\{d_{n}\right\}_{n=1}^{\infty}$ uma sequência encadeada positiva, existe uma única medida de probabilidade não trivial $\mu$ no círculo unitário. Além disso, os coeficientes de Verblunsky $\left\{\alpha_{n}\right\}_{n=0}^{\infty}$, associados aos polinômios ortogonais com respeito a $\mu$, são dados pela relação

$$
\alpha_{n-1}=\bar{\tau}_{n-1}\left[\frac{1-2 m_{n}-i c_{n}}{1-i c_{n}}\right], \quad n \geq 1,
$$

onde $\tau_{0}=1, \tau_{n}=\prod_{k=1}^{n}\left(1-i c_{k}\right) /\left(1+i c_{k}\right), n \geq 1$ e $\left\{m_{n}\right\}_{n=0}^{\infty}$ é a sequência de parâmetros minimal para $\left\{d_{n}\right\}_{n=1}^{\infty}$. Neste trabalho, impondo algumas restrições sobre o par de sequências reais $\left\{\left\{c_{n}\right\}_{n=1}^{\infty},\left\{m_{n}\right\}_{n=1}^{\infty}\right\}$, mostramos que é possível obter medidas de probabilidade não triviais no círculo unitário cujos respectivos coeficientes de Verblunsky são periódicos.

Palavras-chave. Medidas de Probabilidade, Coeficientes de Verblunsky Periódicos, Sequências Encadeadas Positivas, Sequências Periódicas

\section{Introdução}

Nos últimos anos os polinômios ortogonais no círculo unitário, também conhecidos como polinômios de Szegő, vêm recebendo bastante atenção de muitos pesquisadores. Este fato tem ocorrido principalmente em razão de suas aplicações em diversas áreas da Matemática. Regras de quadratura, processamento de sinais e teoria espectral são alguns dos muitos tópicos em que estes polinômios estão inseridos (veja, por exemplo, [1,6]).

\footnotetext{
${ }^{1}$ cleonice@ibilce.unesp.br

2jairo.santos@ufma.br

${ }^{3}$ ranga@ibilce.unesp.br

${ }^{4}$ doveronese@yahoo.com.br
} 
Textos mais recentes sobre tais polinômios podem ser encontrados nos dois volumes de Simon $[7,8]$.

Seja $\mu(z)=\mu\left(e^{i \theta}\right)$ uma medida no círculo unitário $\mathbb{T}=\left\{z=e^{i \theta}: 0 \leq \theta \leq 2 \pi\right\}$. De acordo com a terminologia adotada em Simon [7], a medida $\mu$ é dita não trivial se o seu suporte é um conjunto infinito e $\mu$ é dita uma medida de probabilidade se $\mu(\mathbb{T})=1$.

Se $\mu$ é uma medida de probabilidade não trivial no círculo unitário $\mathbb{T}$, podemos definir a sequência associada de polinômios ortogonais no círculo unitário, $\left\{\phi_{n}\right\}_{n=0}^{\infty}$, por

$$
\int_{\mathbb{T}} \bar{z}^{j} \phi_{n}(z) d \mu(z)=\int_{0}^{2 \pi} e^{-i j \theta} \phi_{n}\left(e^{i \theta}\right) d \mu\left(e^{i \theta}\right)=0, \quad 0 \leq j \leq n-1, \quad n \geq 1 .
$$

Tomando $\kappa_{n}^{-2}=\left\|\phi_{n}\right\|^{2}=\int_{\mathbb{T}}\left|\phi_{n}(z)\right|^{2} d \mu(z)$, os polinômios ortonormais são dados por $\varphi_{n}(z)=\kappa_{n} \phi_{n}(z), n \geq 0$.

Os polinômios ortogonais no círculo unitário na forma mônica satisfazem a relação de recorrência

$$
\phi_{n}(z)=z \phi_{n-1}(z)-\bar{\alpha}_{n-1} \phi_{n-1}^{*}(z) \quad n \geq 1,
$$

onde $\alpha_{n-1}=-\overline{\phi_{n}(0)}$ e $\phi_{n}^{*}(z)=z^{n} \overline{\phi_{n}(1 / \bar{z})}$ denota o polinômio recíproco de $\phi_{n}(z)$.

Os números $\alpha_{n}$ são chamados de coeficientes de Verblunsky. Sabe-se que esses coeficientes são tais que $\left|\alpha_{n}\right|<1(n \geq 0)$ e que, além disso, eles são suficientes para caracterizar completamente tanto os polinômios ortogonais como a medida associada (veja, por exemplo, [7, Teorema 1.7.11]).

Segundo Chihara [3], uma sequência $\left\{a_{n}\right\}_{n=1}^{\infty}$ é dita uma sequência encadeada positiva se existe uma outra sequência $\left\{g_{n}\right\}_{n=0}^{\infty}$ tal que

$$
0 \leq g_{0}<1, \quad 0<g_{n}<1, \text { para } n \geq 1, \quad \text { e } \quad a_{n}=\left(1-g_{n-1}\right) g_{n}, \text { para } n \geq 1 .
$$

A sequência $\left\{g_{n}\right\}_{n=0}^{\infty}$ é chamada de uma sequência de parâmetros para a sequência encadeada positiva $\left\{a_{n}\right\}_{n=1}^{\infty}$ e pode não ser única. Além disso, toda sequência encadeada positiva possui uma sequência de parâmetros minimal, denotada por $\left\{m_{n}\right\}_{n=0}^{\infty}$, obtida com $m_{0}=0$, e uma sequência de parâmetros maximal, denotada por $\left\{M_{n}\right\}_{n=0}^{\infty}$, a qual é caracterizado pela condição de que se $g_{0}>M_{0}$ então $\left\{g_{n}\right\}_{n=1}^{\infty}$ gerada por $g_{n}=a_{n} /\left(1-g_{n-1}\right), n \geq 1$ não satisfaz $0<g_{n}<1, n \geq 1$.

Recentemente, foi mostrado em [4] que dado um par de sequências reais $\left\{\left\{c_{n}\right\}_{n=1}^{\infty}\right.$, $\left.\left\{d_{n}\right\}_{n=1}^{\infty}\right\}$, onde $\left\{d_{n}\right\}_{n=1}^{\infty}$ é uma sequência encadeada positiva, então correspondente a este par, existe uma única medida de probabilidade não trivial no círculo unitário $\mathbb{T}$. Desta forma, também podemos olhar para a medida a partir do par associado de sequências reais $\left\{c_{n}\right\}_{n=1}^{\infty}$ e $\left\{d_{n}\right\}_{n=1}^{\infty}$. No Teorema 2.1 fornecemos uma informação mais completa sobre este resultado e veremos que as sequências de coeficientes de Verblunsky estão diretamente relacionadas com as sequências reais $\left\{c_{n}\right\}_{n=1}^{\infty}$ e $\left\{m_{n}\right\}_{n=1}^{\infty}$, onde $\left\{m_{n}\right\}_{n=0}^{\infty}$ é a sequência de parâmetros minimal para a sequência encadeada positiva $\left\{d_{n}\right\}_{n=1}^{\infty}$ (para mais detalhes sobre sequências encadeadas veja, por exemplo, [3]).

Existem muitos trabalhos na teoria de polinômios ortogonais no círculo unitário que estudam medidas de probabilidade cujas sequências associadas de coeficientes de Verblunsky são $p$-periódicas, isto é, $\alpha_{n+p}=\alpha_{n}, n \geq 0$ e $p \in \mathbb{N}$. Resultados sobre o suporte 
essencial de tais medidas, função peso e possíveis pontos puros (bem como o tamanho da massa, quando existem) são completamente conhecidos. Por exemplo, os possíveis pontos puros dessas medidas são soluções da equação $\varphi_{p}^{*}(z)-\varphi_{p}(z)=0$ (veja [8, Capítulo 11]). Sendo assim, torna-se interessante conhecer medidas de probabilidade não triviais onde a sequência associada de coeficientes de Verblunsky, obtida a partir do par de sequências reais $\left\{\left\{c_{n}\right\}_{n=1}^{\infty},\left\{m_{n}\right\}_{n=1}^{\infty}\right\}$, é periódica.

Um fato importante é que podemos ter uma medida cujos coeficientes de Verblunsky são periódicos e tal que as sequências reais associadas, $\left\{c_{n}\right\}_{n=1}^{\infty}$ e $\left\{m_{n}\right\}_{n=1}^{\infty}$, não são periódicas (veja, por examplo, [5]). Além disso, também é possível obter sequências reais $\left\{c_{n}\right\}_{n=1}^{\infty}$ e $\left\{m_{n}\right\}_{n=1}^{\infty}$ periódicas sem que a medida associada possua seus respectivos coeficientes de Verblunsky periódicos.

O objetivo deste trabalho é estudar medidas de probabilidade não triviais no círculo unitário, onde a sequência associada de coeficientes de Verblunsky é periódica e obtida por meio de um par de sequência reais periódicas $\left\{\left\{c_{n}\right\}_{n=1}^{\infty},\left\{m_{n}\right\}_{n=1}^{\infty}\right\}$, com uma restrição de sinal sobre a sequência $\left\{c_{n}\right\}_{n=1}^{\infty}$.

\section{Resultado Preliminar}

Apresentamos inicialmente um resultado (estabelecido em [4]) que fornece uma caracterização para medidas de probabilidade não triviais em termos de duas sequências reais.

Teorema 2.1. (a) Dada uma medida de probabilidade não trivial $\mu$ sobre o círculo unitário, então associado a esta, existe um único par de sequências reais $\left\{\left\{c_{n}\right\}_{n=1}^{\infty},\left\{d_{n}\right\}_{n=1}^{\infty}\right\}$, onde $\left\{d_{n}\right\}_{n=1}^{\infty}$ é também uma sequência encadeada positiva. Especificamente, se $\left\{\alpha_{n}\right\}_{n=0}^{\infty}$ é a sequência associada de coeficientes de Verblunsky e se a sequência $\tau_{n}$ é tal que $\tau_{0}=1$ e $\tau_{n}=\tau_{n-1}\left(1-\bar{\tau}_{n-1} \bar{\alpha}_{n-1}\right) /\left(1-\tau_{n-1} \alpha_{n-1}\right), n \geq 1$, então $m_{0}=0$,

$$
c_{n}=\frac{-\operatorname{Im}\left(\tau_{n-1} \alpha_{n-1}\right)}{1-\operatorname{Re}\left(\tau_{n-1} \alpha_{n-1}\right)} \quad \text { e } \quad m_{n}=\frac{1}{2} \frac{\left|1-\tau_{n-1} \alpha_{n-1}\right|^{2}}{\left[1-\operatorname{Re}\left(\tau_{n-1} \alpha_{n-1}\right)\right]}, \quad n \geq 1,
$$

onde $\left\{m_{n}\right\}_{n=0}^{\infty}$ é a sequência de parâmetros minimal de $\left\{d_{n}\right\}_{n=1}^{\infty}$. Além disso, a sequência de parâmetros maximal $\left\{M_{n}\right\}_{n=0}^{\infty}$ de $\left\{d_{n}\right\}_{n=1}^{\infty}$ é tal que $M_{0}$ é o valor do salto na medida em $z=1$.

(b) Reciprocamente, dado um par de sequências reais $\left\{\left\{c_{n}\right\}_{n=1}^{\infty},\left\{d_{n}\right\}_{n=1}^{\infty}\right\}$, onde $\left\{d_{n}\right\}_{n=1}^{\infty}$ é também uma sequência encadeada positiva, então, associado a este par, existe uma única medida de probabilidade não trivial $\mu$ com suporte no círculo unitário. Especificamente, se $\left\{m_{n}\right\}_{n=0}^{\infty}$ é a sequência de parâmetros minimal de $\left\{d_{n}\right\}_{n=1}^{\infty}$, então $\tau_{0}=1$,

$$
\tau_{n-1} \alpha_{n-1}=\frac{1-2 m_{n}-i c_{n}}{1-i c_{n}} \quad e \quad \tau_{n}=\frac{1-i c_{n}}{1+i c_{n}} \tau_{n-1}, \quad n \geq 1 .
$$

Além disso, a medida tem um salto $M_{0}$ em $z=1$, onde $\left\{M_{n}\right\}_{n=0}^{\infty}$ é a sequência de parâmetros maximal de $\left\{d_{n}\right\}_{n=1}^{\infty}$. 


\section{Medidas com Coeficientes de Verblunsky Periódicos}

O primeiro resultado desta seção fornece uma caracterização para medidas com sequência associada de coeficientes de Verblunsky periódica em termos de um par de sequências reais $\left\{\left\{c_{n}\right\}_{n=1}^{\infty},\left\{d_{n}\right\}_{n=1}^{\infty}\right\}$, onde $\left\{d_{n}\right\}_{n=1}^{\infty}$ é uma sequência encadeada positiva. Ao longo desta seção, faremos $b_{n}=1-2 m_{n}, n \geq 1$, onde $\left\{m_{n}\right\}_{n=0}^{\infty}$ é a sequência de parâmetros minimal de $\left\{d_{n}\right\}_{n=1}^{\infty}$.

Lema 3.1. Seja $\mu$ a medida de probabilidade associada ao par de sequências reais $\left\{\left\{c_{n}\right\}_{n=1}^{\infty}\right.$, $\left.\left\{d_{n}\right\}_{n=1}^{\infty}\right\}$ como no Teorema 2.1. Então, a medida $\mu$ possui sequência associada de coeficientes de Verblunsky, $\left\{\alpha_{n}\right\}_{n=0}^{\infty}, p$-periódica se, e somente se, para $n \geq 0$,

$$
\sum_{j=n+1}^{n+p} \arg \left(\frac{1+i c_{j}}{1-i c_{j}}\right)=\arg \left(\frac{b_{n+1}-i c_{n+1}}{1-i c_{n+1}}\right)-\arg \left(\frac{b_{n+p+1}-i c_{n+p+1}}{1-i c_{n+p+1}}\right)+2 k_{n} \pi, k_{n} \in \mathbb{Z}
$$

$e$

$$
\frac{b_{n+1}^{2}+c_{n+1}^{2}}{1+c_{n+1}^{2}}=\frac{b_{n+p+1}^{2}+c_{n+p+1}^{2}}{1+c_{n+p+1}^{2}} .
$$

Demonstração. Primeiro, usando (1), observamos que, para $n \geq 0, \alpha_{n+p}=\alpha_{n}$ se, e somente se,

$$
\bar{\tau}_{n+p}\left[\frac{b_{n+p+1}-i c_{n+p+1}}{1-i c_{n+p+1}}\right]=\bar{\tau}_{n}\left[\frac{b_{n+1}-i c_{n+1}}{1-i c_{n+1}}\right]
$$

ou, equivalentemente,

$$
\left[\prod_{j=n+1}^{n+p} \frac{1+i c_{j}}{1-i c_{j}}\right]\left[\frac{b_{n+p+1}-i c_{n+p+1}}{1-i c_{n+p+1}}\right]=\left[\frac{b_{n+1}-i c_{n+1}}{1-i c_{n+1}}\right] .
$$

Agora, o resultado segue comparando-se os módulos e os argumentos dos números

$$
\left[\prod_{j=n+1}^{n+p} \frac{1+i c_{j}}{1-i c_{j}}\right]\left[\frac{b_{n+p+1}-i c_{n+p+1}}{1-i c_{n+p+1}}\right] \text { e }\left[\frac{b_{n+1}-i c_{n+1}}{1-i c_{n+1}}\right], n \geq 0 .
$$

Observação 3.1. Dizemos que uma medida de probabilidade no círculo unitário $\mu$ é uma medida simétrica se $d \mu(z)=-d \mu(1 / z), z \in \mathbb{T}$. Resultados estabelecidos em [2] garantem que $\mu$ é uma medida simétrica se, e somente se, $c_{n}=0, n \geq 1$, onde a sequência real $\left\{c_{n}\right\}_{n=1}^{\infty}$ é dada como no Teorema 2.1.

Teorema 3.1. Seja $\mu$ a medida de probabilidade associada ao par de sequências reais $\left\{\left\{c_{n}\right\}_{n=1}^{\infty},\left\{d_{n}\right\}_{n=1}^{\infty}\right\}$ como no Teorema 2.1. Suponha que $\left\{c_{n}\right\}_{n=1}^{\infty}$ e $\left\{m_{n}\right\}_{n=1}^{\infty}$ são periódicas de período $p$ e que a sequência $\left\{c_{n}\right\}_{n=1}^{\infty}$ satisfaz $c_{2 n}=-c_{2 n-1}$, para $n \geq 1$. Então,

(i) Se p é par, a medida $\mu$ possui coeficientes de Verblunsky p-periódicos; 
(ii) Se p é ímpar, a medida $\mu$ possui coeficientes de Verblunsky p-periódicos se, e somente se, $\mu$ é simétrica.

Demonstração. (i) Usando as hipóteses satisfeitas pelo par de sequências reais $\left\{\left\{c_{n}\right\}_{n=1}^{\infty}\right.$, $\left.\left\{m_{n}\right\}_{n=1}^{\infty}\right\}$ é fácil ver que (2) e (3) ocorrem. Logo, o resultado segue pelo Lema 3.1.

(ii) Se $p$ é ímpar, fazendo $n=0$ em (2) e usando novamente as hipóteses satisfeitas pelas sequências $\left\{c_{n}\right\}_{n=1}^{\infty}$ e $\left\{m_{n}\right\}_{n=1}^{\infty}$ podemos ver que $\arg \left(\frac{1+i c_{p}}{1-i c_{p}}\right)=2 \tilde{k}_{p} \pi, \tilde{k}_{p} \in \mathbb{Z}$. Consequentemente, $c_{p}=0$ e também $c_{p+1}=-c_{p}=0$. Agora, usando a periodicidade de $\left\{c_{n}\right\}_{n=1}^{\infty}$ e a hipótese que $c_{2 n}=-c_{2 n-1}$, concluímos que $c_{n}=0$, para todo $n \geq 1$. Portanto, $\mu$ é simétrica (veja Observação 3.1). Reciprocamente, se $\mu$ é simétrica, então $c_{n}=0$, para $n \geq 1$. Daí, as condições (2) e (3) do Lema 3.1 podem ser facilmente verificadas, de onde concluímos que a medida $\mu$ possui coeficientes de Verblunsky $p$-periódicos.

O próximo resultado fornece uma caracterização geométrica para a escolha de $\left\{c_{n}\right\}_{n=1}^{\infty}$ e $\left\{m_{n}\right\}_{n=1}^{\infty}$ considerada no teorema anterior.

Teorema 3.2. Seja p um número natural par e $\mu$ a medida de probabilidade associada ao par de sequências reais $\left\{\left\{c_{n}\right\}_{n=1}^{\infty},\left\{d_{n}\right\}_{n=1}^{\infty}\right\}$ como no Teorema 2.1. Então, as seguintes afirmações são equivalentes.

(i) As sequências $\left\{c_{n}\right\}_{n=1}^{\infty}$ e $\left\{m_{n}\right\}_{n=1}^{\infty}$ são $p$-periódicas, com $c_{2 n}=-c_{2 n-1}, n \geq 1$.

(ii) A sequência de coeficientes de Verblunsky, $\left\{\alpha_{n}\right\}_{n=0}^{\infty}$, associada com a medida $\mu$ é $p$-periódica. Além disso, para cada $k \in\{0,1, \ldots,(p-2) / 2\}$, as retas $r_{2 k}$, passando por $\alpha_{2 k}$ e 1 , e $r_{2 k+1}$, passando por $\alpha_{2 k+1} e-1$, são paralelas.

Demonstração. (i) $\Rightarrow$ (ii) Pelo Teorema 3.1 é imediato que $\left\{\alpha_{n}\right\}_{n=0}^{\infty}$ é uma sequência periódica com período $p$. Além disso, usando (1) e a hipótese que $c_{2 n}=-c_{2 n-1}$, temos

$$
\alpha_{2 n}=1+\lambda_{2 n}\left(-1-i c_{2 n+1}\right) \quad \text { e } \quad \alpha_{2 n+1}=-1+\lambda_{2 n+1}\left(-1+i c_{2 n+2}\right), \quad n \geq 0,
$$

onde $\lambda_{2 n}=\left(1-b_{2 n+1}\right) /\left(1+c_{2 n+1}^{2}\right)$ e $\lambda_{2 n+1}=-\left(1+b_{2 n+2}\right) /\left(1+c_{2 n+2}^{2}\right)$.

Portanto, para cada $k \in\{0,1, \ldots,(p-2) / 2\}$, podemos ver que $\alpha_{2 k} \in r_{2 k}$ e $\alpha_{2 k+1} \in$ $r_{2 k+1}$, onde $r_{2 k}$ e $r_{2 k+1}$ são retas com equações paramétricas dadas, respectivamente, por $r_{2 k}(t)=1+t\left(-1-i c_{2 k+1}\right)$ e $r_{2 k+1}(t)=-1+t\left(-1+i c_{2 k+2}\right), t \in \mathbb{R}$.

Finalmente, como para cada $k \in\{0,1, \ldots,(p-2) / 2\}$ temos, por hipótese, $c_{2 k+1}=$ $-c_{2 k+2}$, então $-1-i c_{2 k+1}=-1+i c_{2 k+2} \mathrm{e}$, consequentemente, $r_{2 k} \| r_{2 k+1}$.

(ii) $\Rightarrow$ (i) Seja $\alpha_{j}=x_{j}+i y_{j}, j \geq 0$. Se $j=2 s, s \geq 0$, podemos escrever

$$
\alpha_{2 s}=1+\lambda_{2 s}\left(-1-i \tilde{c}_{2 s+1}\right),
$$

onde

$$
\lambda_{2 s}=\frac{1-\tilde{b}_{2 s+1}}{1+\tilde{c}_{2 s+1}^{2}}, \quad \tilde{c}_{2 s+1}=\frac{y_{2 s}}{x_{2 s}-1} \quad \text { e } \quad \tilde{b}_{2 s+1}=1+\frac{\left(x_{2 s}-1\right)^{2}+y_{2 s}^{2}}{x_{2 s}-1} .
$$

Da mesma forma, se $j=2 s+1, s \geq 0$, podemos também escrever

$$
\alpha_{2 s+1}=-1+\lambda_{2 s+1}\left(-1+i \tilde{c}_{2 s+2}\right),
$$


onde

$$
\lambda_{2 s+1}=-\frac{1+\tilde{b}_{2 s+2}}{1+\tilde{c}_{2 s+2}^{2}}, \quad \tilde{c}_{2 s+2}=\frac{-y_{2 s+1}}{1+x_{2 s+1}} \quad \text { e } \quad \tilde{b}_{2 s+2}=-1+\frac{\left(1+x_{2 s+1}\right)^{2}+y_{2 s+1}^{2}}{1+x_{2 s+1}} .
$$

Portanto, fazendo $\tilde{m}_{n}=\left(1-\tilde{b}_{n}\right) / 2$, usando a hipótese que $\alpha_{n+p}=\alpha_{n}, n \geq 0$ e usando os resultados obtidos de (5)-(7), não é difícil ver que

$$
\tilde{c}_{n+p}=\tilde{c}_{n} \quad \text { e } \quad \tilde{m}_{n+p}=\tilde{m}_{n}, \quad n \geq 1 .
$$

Agora, para cada $k \in\{0,1, \ldots,(p-2) / 2\}$, seja $r_{2 k}$ a reta passando por $\alpha_{2 k}$ e 1 , e $r_{2 k+1}$ a reta passando por $\alpha_{2 k+1}$ e -1 . Então, usando (4), (6), (8) e a hipótese que $r_{2 k} \| r_{2 k+1}, k \in\{0,1, \ldots,(p-2) / 2\}$, podemos concluir que

$$
\tilde{c}_{2 n+2}=-\tilde{c}_{2 n+1}, \quad n \geq 0 .
$$

Consequentemente, de (4)-(9), obtemos

$$
\alpha_{2 s}=\frac{\tilde{b}_{2 n+1}+i \tilde{c}_{2 n+2}}{1+i \tilde{c}_{2 n+2}} \quad \text { e } \quad \alpha_{2 s+1}=\frac{\tilde{b}_{2 n+2}-i \tilde{c}_{2 n+2}}{1+i \tilde{c}_{2 n+2}}, \quad s \geq 0 .
$$

Finalmente, usando (1) e as relações obtidas em (10) para $\alpha_{n}$, podemos verificar, por indução matemática, que $\tilde{c}_{n}=c_{n}$ e $\tilde{m}_{n}=m_{n}, n \geq 1$, e isto completa a prova.

\section{Conclusões}

Uma vez que cada medida de probabilidade não trivial (com suporte no círculo unitário) pode ser caracterizada tanto por uma sequência de coeficientes de Verblunsky $\left\{\alpha_{n}\right\}_{n=0}^{\infty}$ quanto por um par de sequências reais $\left\{\left\{c_{n}\right\}_{n=1}^{\infty},\left\{m_{n}\right\}_{n=1}^{\infty}\right\}$, conclui-se que é possível obter, a partir deste par, medidas no círculo unitário cuja respectiva sequência de coeficientes de Verblunsky é $p$-periódica. Para isso, é suficiente impor a restrição de sinal $c_{2 n}=-c_{2 n-1}$ $(n \geq 1)$ e uma $p$-periodicidade nas sequências $\left\{c_{n}\right\}_{n=1}^{\infty}$ e $\left\{m_{n}\right\}_{n=1}^{\infty}$, quando $p$ é par. Além disso, quando $p$ é ímpar, é suficiente considerar $c_{n}=0(n \geq 1)$ e $\left\{m_{n}\right\}_{n=1}^{\infty} p$-periódica.

\section{Agradecimentos}

Os autores agradecem à CAPES, CNPq e FAPESP, pelo apoio financeiro para a realização deste trabalho.

\section{Referências}

[1] J. Breuer, E. Ryckman and B. Simon, Equality of the spectral and dynamical definitions of reflection, Comm. Math. Phys., 295:531-550, 2010. 
[2] K. Castillo, M. S. Costa, A. Sri Ranga and D. O. Veronese, A Favard type theorem for orthogonal polynomials on the unit circle from a three term recurrence formula, J. Approx. Theory, 184:146-162, 2014.

[3] T. S. Chihara, An Introduction to Orthogonal Polynomials, Mathematics and its Applications Series, Gordon and Breach, New York, 1978.

[4] M. S. Costa, H. M. Felix and A. Sri Ranga, Orthogonal polynomials on the unit circle and chain sequences, J. Approx. Theory, 173:14-32, 2013.

[5] M. S. Costa, R. L. Lamblém, J. H. McCabe and A. Sri Ranga, Para-orthogonal polynomials from constant Verblunsky coefficients, J. Math. Anal. Appl., 426:10401060, 2015.

[6] F. Peherstorfer, Positive trigonometric quadrature formulas and quadrature on the unit circle, Math. Comp., 80:1685-1701, 2011.

[7] B. Simon, Orthogonal Polynomials on the Unit Circle. Part 1. Classical Theory, Amer. Math. Soc. Colloq. Publ., vol. 54, part 1, Providence, RI, 2005.

[8] B. Simon, Orthogonal Polynomials on the Unit Circle. Part 2. Spectral Theory, Amer. Math. Soc. Colloq. Publ., vol. 54, part 2, Providence, RI, 2005. 